\title{
The Same or Different? Investigating Whether Trust and Distrust Are Orthogonal Constructs or Span a Continuum
}

\author{
August Capiola \\ Air Force Research Laboratory \\ Wright-Patterson AFB, OH \\ august.capiola.1@us.af.mil \\ Sarah A. Jessup \\ Air Force Research Laboratory \\ Wright Patterson AFB, OH \\ sarah.jessup.ctr@us.af.mil
}

\author{
Gene M. Alarcon \\ Air Force Research Laboratory \\ Wright-Patterson AFB, OH \\ gene.alarcon.1@us.af.mil
}

\author{
Anthony M. Gibson \\ Consortium of Universities, \\ Washington, DC \\ gibson.88@,wright.edu \\ Izz Aldin Hamdan \\ General Dynamics Information \\ Technology \\ Dayton, $\mathrm{OH}$ \\ izzy.hamdan@gdit.com
}

\begin{abstract}
Trust has been investigated across many psychology sub-disciplines. However, there is a debate in the literature as to whether a) trust is a continuum ranging from trust to distrust or b) if trust and distrust are orthogonal constructs. The present research investigated these postulates by assessing selfreported measures of trust and distrust before and after an experimental task. Participants engaged in a trust game and were randomized to experience trust or distrust behaviors across several trials. The results showed that self-reported measures of trust and distrust were highly correlated. Moreover, the experimental manipulation evidenced comparable effects on both trust and distrust criterion. The results support the postulate that trust and distrust compose a continuum, with trust at one end and distrust at the other. Practically speaking, researchers may wish to simply assess a measure of trust rather than assessing multiple self-report measures of the same construct (i.e., trust and distrust).
\end{abstract}

\section{Introduction}

Trust is an important interpersonal construct and has provided fodder for research since the late 1950s [see 1]. Twenty-five years ago, a review of the extant literature led to an explication of the trust process [2]. Therein, Mayer et al. defined trust as "the willingness of a party to be vulnerable to the actions of another party based on the expectation that the other will perform a particular action important to the trustor, irrespective of the ability to monitor or control the other party" (p. 712). From this seminal article, researchers have shown that Mayer and colleagues' postulates are both relevant and model the trust process in person-to-person [3] and team [4] contexts. Furthermore, these postulates have been applied to distributed teaming contexts [5] as well as trust toward automation [6] and robot contexts [7].

Shortly after [2]'s seminal paper, Lewicki et al. published their conceptualization of the trust process [8]. Though this model had several similarities to that of [2]'s model, [8] explicitly postulated that trust and distrust are orthogonal. That is, trust and distrust are distinct constructs. Lewicki and colleagues cited several examples from the neighboring literatures on affect [9] and attitudes [10] to support their model of trust and distrust as orthogonal constructs. Nearly a decade later, Schoorman et al. [11] responded to the postulates of [8], arguing that rather trust spans a continuum ranging from trust to distrust [11].

Both of these groups of researchers [2; 8 ; see also 11] have theoretically argued for their model of trust and have cited empirical research claiming it supports their postulates. However, except for a few studies, which have cited the discrepancies between the models $[12 ; 13]$, the authors of the present research are not aware of any research that has directly tested the models of both [2] and [8]. That is, the authors are unaware of an experiment that has investigated whether trust and distrust are represented on a continuum or as two orthogonal constructs. In the current paper, the assumptions of both models are reviewed along with relevant citations which bolster the respective groups' arguments. We present an experiment which aimed to directly compare whether trust is more usefully measured as a continuum or as an orthogonal construct to distrust.

Trust research has implications for the multidisciplinary fields of system sciences and 
information systems $[14 ; 15]$, particularly computermediated interaction (e.g., [16, 17]). As interactions become more distributed and teams of teams become more heterogeneous in their composition, understanding when and why trust impacts performance outcomes will continue to increase in importance (e.g., [18]). As such, having an appropriate conceptualization and measurement of the trust construct will aid researchers in testing hypotheses pertaining to trust and uncovering how it influences interactions in a multitude of contexts.

\section{Related Work}

\subsection{Two Prominent Models of Interpersonal Trust}

Mayer and colleagues' [2] formative paper explicates the trust process. That is, the willingness to accept vulnerability (i.e., trust) has both antecedents and consequences. Antecedents to trust are both statedependent and trait-based. As characteristics of the referent help to determine whether a trustor will accept vulnerability, [2] postulated three main antecedents to trust that are indicative of the trustor's perceptions of the referent. These characteristics of the referent are ability, benevolence, and integrity. Ability concerns the trustor's perceptions of the referent's competence and capabilities to do something relevant to the trustor. Benevolence concerns the perceptions the trustor has about the referent having their best interest in mind outside of the referent's own egocentric motivations. Integrity concerns the perceptions that the referent possesses principles and ethics relevant to the interchange that the trustor finds acceptable. In addition, propensity to trust is a willingness to trust others in general. This generalized expectation of and willingness to accept vulnerability from others is considered to shape trust early on in an interchange when a trustor knows little about the referent [see also 19; 20]. Together, these state- and trait-based antecedents help to predict whether the trustor will trust the referent $[2 ; 11]$. The antecedents may be differently influential on trust; for instance, a referent may be perceived as competent in their ability to accomplish that which the trustor holds to be important, but if the trustor does not perceive the referent to be ethical or have their best interest in mind, the trustor may not be willing to trust them. Together, these antecedents predict one's willingness to trust, which is an intention to be vulnerable.

Researchers have utilized [2]'s model of trust to guide their research across a litany of studies. Jarvenpaa and colleagues [5] found that perceptions of a referent's integrity and ability, along with the trustor's propensity to trust (i.e., the general willingness to accept vulnerability toward others), were related to trust early on in an interchange between ad hoc distributed teammates. Later, however, perceptions of benevolence were related to trust. In a meta-analysis, Colquitt et al. [3] showed that [2]'s model of trust is relevant to predicting workplace behaviors. Specifically, perceptions of ability, benevolence, and integrity, as well as propensity to trust, are predictive of trust and some performance outcomes, and that trust is directly related to performance outcomes. However, [2]'s conceptualization of trust is described as a continuum of accepting vulnerability. Specifically, not only were the trustworthiness antecedents as well as propensity to trust postulated to span a continuum, so too was the willingness to be vulnerable to a referent [see also 11]. Around the same time as [2]'s initial work, [8] published their paper on the trust process, which we will turn to next.

Lewicki et al. [8], similar to [2], postulated that trust may be a willingness to accept vulnerability. However, [8] believe that distrust - a confident negative expectation regarding another's conduct - is theoretically orthogonal from trust. Specifically, [8]'s central argument resides with the suppositions of ambivalence. Lewicki and colleagues cite literature about individuals that can have positive and negative attitudes toward quitting smoking [21], particular races [22], and job satisfaction [23]. In all of these examples, [8] make the central assumptions that just as positive and negative affect can be ascribed to a referent, so too can trust and distrust. Take the example in which a person may want to purchase a bike. The individual may want to exercise, become healthier, and have a more eco-friendly way to get to and from work. However, the individual may also feel hesitant because increasing their biking frequency may not be the safest mode of transportation, and they do not want to spend money on a new mode of transportation. Thus, the individual exhibits ambivalence toward the bike purchase. Just as a person may attribute this ambivalence to a referent, so too can a trustor ascribe ambivalence toward a trustee.

However, it should be noted that although [8] argue that trust and distrust are orthogonal, they stated that this theory should be empirically studied to determine the relationship between the two constructs.

\subsection{Trust and Distrust: A Continuum or Orthogonal Constructs?}

Schoorman et al. [11] argued that in contrast to the postulates of [8], trust is not orthogonal to distrust but rather spans a continuum from trust to distrust. 
Schoorman and colleagues [11] cite the definition of trust and deduce that the absence of trust is distrust. Specifically, they cite both the Webster's and Random House dictionary definitions, which defined distrust as a lack/absence of trust or to have no trust, respectively, along with definitions from sociology, which convey similarly that distrust is to have no trust. The authors also argue that often people may ascribe differential perceptions of trustworthiness toward referents depending on the attribute of the referent that they are focusing. For instance, a trustor may find a colleague to have sound investment judgement in their financial decisions for a company at work. However, that same colleague may have poor judgement in managing their personal funds. Thus, the trustor may perceive the colleague has integrity in their financial dealings in the workplace but not in their personal finances. What [8] postulate as ambivalence is actually perceptions of trustworthiness toward the referent in two different contexts (i.e., the colleague's financial integrity in the workplace and their personal financial integrity). From this, trust antecedents as well as intentions to be vulnerable (i.e., trust), reside along a continuum. Theoretically, [11] — expanding [2]'s initial theories to directly respond to [8] — provide a deductive argument that trust resides along a continuum with distrust at one end and trust at the other end. The space between these two extremes characterizes variability in the degree to which a trustor trusts a referent.

Recently, researchers have begun to investigate whether state-based measures of trust and distrust are unique constructs. Indeed, Lyons et al. [24] conducted an exploratory factor analysis and demonstrated trust and distrust emerged as unique constructs. Trust and distrust predicted differential variance in criterion (e.g., participants' decision confidence toward their route selection in a simulated military convoy task). However, [24] may have observed two distinct factors that related to item valence (i.e., positive or negative) rather than two trust factors. Thus, more research is needed to determine the exact nature of trust and distrust.

McKnight and Choudhury [25] explored trust and distrust in a business to consumer electroniccommerce setting. They tested whether trust and distrust demonstrated discriminate validity and if trust and distrust differed in their predictions of willingness to use a fictional website. In their study, participants were presented with a (fictional) website that provided legal advice and were asked to rate their (dis)trust intention in the website, willingness to follow website advice, and other characteristics of the websites. They found trust intentions were a stronger predictor of risktaking behavior (i.e., following a website's advice) than were distrust intentions. A hypothesis test found that the positive correlation between trust intentions and following the websites advice was significantly greater than the negative correlation between distrust intentions and following the websites advice. As such, [25] propose that trust and distrust are orthogonal and should be measured separately. However, their measure of willingness to follow the website overlaps with [2]'s definition of trust, which is a willingness to be vulnerable. Therefore, the findings from their study should be interpreted with caution.

Mittendorf [26] investigated the relationship between trust and distrust in a rideshare context. Specifically, participants were asked to rate their trust and distrust in a ridesharing platform, as well as the rideshare drivers. In addition, participants rated their intentions to engage and request a ride from the particular rideshare platform. Trust and distrust toward both rideshare and driver referents were moderately (negatively) correlated. In addition, trust toward the platform (but not the driver) and distrust toward the driver (but not the platform) were (negatively) correlated with intentions to engage. As such, the researcher concluded that trust and distrust emerged as separate constructs. This finding aligns with [8]'s argument that trust and distrust are orthogonal factors that should be assessed separately. However, [26] may have conflated measures of trust and distrust and their intentions to engage variables. Specifically, the intentions to engage variable could be considered trust as per definitions from [2] and the instruments from Mayer and Davis [27], i.e., willingness (or intentions) to be vulnerable. In addition, [26]'s measures of trust and distrust, though carefully separating referents of the platform and driver (see also [11]), conflate trustworthiness and trust constructs explicated by [2]. Therefore, caution should be exercised when interpreting these results.

More recently, Saunders et al. [13] utilized card sort methodologies and interview techniques to ascertain whether individuals working in two different contexts ascribe differential trust and distrust toward workplace change experiences (i.e., positively and negatively perceived organizational restructuring). They asked participants to rate their emotions toward 49 different categories - several of which were outlined in [8] as pertaining to trust and distrust-in reference to the organizational changes. Participants rated their emotions on a 4-category ordinal scale from 'do not feel' (coded as 'low') to 'feel to some extent (coded as 'weak'), 'feel strongly,' and 'feel most strongly' (both coded as 'high'). This card sort was followed up with in-depth interviews asking participants to elaborate on their selections. Saunders et al. [13] found that some individuals did in fact ascribe feelings of differential trust and distrust toward 
a workplace referent, particularly in feelings of low trust and high distrust (i.e., high and weak distrust characterizations), high trust and low distrust (i.e., high and weak trust characterizations), and low trust and low distrust (i.e., absence of both trust and distrust). These results support [8]'s postulates that trust and distrust can co-occur. Importantly, however, [13] found only one instance in which a participant described a scenario laden with both high trust and high distrust toward the workplace, and even then, the participant described only feeling this to some extent (i.e., "weak"). Saunders and colleagues interpreted this as arguably [8]'s most important example of ambivalence as being practically absent in their data. Moreover, [13] found that in their total sample $(N=$ 56), 44 participants felt either trust or distrust toward a workplace change referent without feeling the opposite, which the researchers interpret as considerable support for [11]'s postulate that trust comprises a continuum. Still, [13] do show some evidence for conceptualizations of trust proposed by both [8] and [2], as well as [11]. As such, [13] called for more research into this debate, and this is exactly the purpose of the present research, which includes using validated scale measures and examining the effects of trust manipulations on two criteria.

\subsection{The Present Research}

Researchers $[2 ; 11]$ have argued that trust spans a continuum ranging from trust to distrust. For [2] and [11], it is illogical to assume that trust and distrust could co-occur. In contrast, [8] argue that trust is an orthogonal construct to distrust such that each are unique and could emerge simultaneously. Regardless of which perspective is adopted, testing these postulates through an analytic approach is needed. To the authors' knowledge, no study has investigated whether measures of trust and distrust are measurably distinct or simply represent the same trust continuum. The current study aims to do this by assessing selfreport measures of trust and distrust before and after a task, which comprised a trust manipulation (see Method section below). We assessed the correlation between the two measures before the trust manipulation, after the trust manipulation, and then assessed the effect of the trust manipulation on selfreported trust toward the referent, self-reported distrust toward the referent, and compare those effect sizes. With this strategy, we assessed whether trust is a measurably distinct construct from distrust, and practically speaking, whether it is relevant to assess trust and distrust with two separate measures. Based on the postulates of both $[2 ; 11]$ and $[8]$, we outline two hypotheses and one research question below:
$H_{1}$ : Self-report assessments of trust and distrust will be significantly, negatively correlated before a trust manipulation.

$\mathrm{H}_{2}$ : Self-report assessments of trust and distrust will be significantly, negatively correlated after a trust manipulation.

$R Q_{1}$ : Does a trust manipulation have differential effects on self-reported trust and distrust scales?

\section{Method}

\subsection{Participants}

Participants $(N=67)$ were adults recruited from a university in the Midwest. They were randomly distributed among two experimental conditions: Trust $(n=33)$ and Distrust $(n=34)$. Ages ranged between 18 and 32 years $(M=21.75, S D=3.70)$. About half $(51 \%)$ were female and white $(40 \%)$. Participants were recruited from the Introduction to Psychology participant pool, flyers, email, and word of mouth. Participants received compensation (i.e., a \$30 gift card) for their participation, as well as cash for all money they earned in the task. The study was approved and overseen by an institutional review board.

\subsection{Task}

The task that participants played over the course of the experiment was Checkmate [28]. Checkmate is a computer game played usually between two players. It is a modified version of the trust (or investor/dictator) game [29]. In this study, the participant was always assigned the role of the "Banker" (investor in the investment/dictator game), and a confederate played the role of the "Runner" (dictator in the investor/dictator game). The role of the Banker was to loan money to the Runner over the course of five rounds. The role of the Runner was to collect boxes in a virtual maze over the course of five rounds. The number of boxes collected by the Runner reflected performance. The initial amount of money in the Banker's virtual account was set at $\$ 50$. The Banker loaned money to the Runner each round in anticipation of earning interest on their investment. Each round, the Banker chose to loan one of three amounts to the Runner: small (\$1-\$7), medium (\$4$\$ 10)$, or large (\$7-\$13). Based on their selections, a pre-determined algorithm specified the exact dollar amount that would be sent to the Runner. 
The Runner chose a risk level for the purpose of potentially increasing the initial loan amount. The risk levels were low $(75-150 \%)$, moderate $(50-200 \%)$, and high (0-300\%). The Runner could earn more money by choosing a higher risk level, but the Runner risked not earning any money at all if his performance was poor. If the Runner decided to err on the side of caution and chose a low risk level, the maximum amount of money the Runner lost was $25 \%$ without collecting any boxes or gained $50 \%$ by performing well.

At the beginning of the round, the Runner chose a risk level. The Runner then promised to return the initial loan and $50 \%$ of the earnings to the Banker. The Banker was notified via a pop-up message which risk level the Runner selected as well as how much of the invested money the Runner promised to return. At this point in the round, the Banker selected an amount to loan to the Runner. Money was then transferred into the Runner's virtual wallet. The maze-running task began, and the Banker was able to watch a top-down video of the Runner's progress. The Runner was allotted two minutes to collect as many boxes as possible. After the maze-running task was over, the Runner then decided how much money to return to the Banker. The Banker received a pop-up message of the exact amount of money the Runner decided to return. In the Trust condition, the amount returned to the Banker was within the range of what the Runner had promised for all time points. In the Distrust condition, the return amount was lower than promised in two of the time points. These steps outlined above were repeated over five rounds. Participants were informed that the amount of money the Banker had in his/her virtual bank at the end of the session belonged to the Banker, and the earnings were paid out in the form of cash, rounded up to the nearest quarter.

\subsection{Experimental Control and Manipulations}

Typically, Checkmate [28] is played between two people. For this study, the participant was always the Banker, and the Runner was a male confederate. The Runner's risk level in the game was set to medium-risk for every round. All of the Runner's data, including maze performance and returning of investment to the Banker, was prerecorded. This level of control allowed our team to focus on the way that participants trusted their partner. However, participants were led to believe they were playing in real-time with the human confederate.

Participants were randomly assigned to one of two experimental conditions: Trust or Distrust. In the Trust condition, the Runner always returned the amount of money that was promised for all rounds. In the Distrust condition, the Runner returned less money than he promised for rounds 3 and 4.

\subsection{Self-Report Measures}

3.4.1. Trust. Trust was measured with the Trust Intentions Scale [27]. This 4-item scale measures a person's willingness or intention to trust others. Participants were asked to use a 5-point agreement response scale ranging from 1 (strongly disagree) to 5 (strongly agree) to describe how much they agreed or disagreed with each statement. The measure was adapted so that the referent in each item was "the Runner." To score, the responses were averaged across participants. A sample item included "I would be willing to let the runner have complete control over my future in this game."

3.4.2. Distrust. Distrust was measured using the Distrust Scale (Adapted from [30]), which is an 8-item scale designed to measure a person's general distrust toward others. The measure was adapted so that the referent in each item was "the Runner." We adapted and reduced the scale to seven items to ensure questions a) were not double loaded and b) were not off base in referencing the state-dependent nature of the referent. Participants were asked to use a 5-point agreement response scale ranging from 1 (strongly disagree) to 5 (strongly agree) to describe how much they agreed or disagreed with each statement. To score, the responses were averaged across participants. A sample item included "The runner will break rules when they have the chance."

\subsection{Procedure}

Participants were run individually in a laboratory comprising two rooms. Participants were introduced to each other once they entered the lab and were then seated in separate rooms. After providing informed consent, participants completed demographic surveys. Then, they completed an endowment earning task, which consisted of five, medium-difficulty, multiple choice math problems. The purpose of this task was to make participants feel like they earned the money used in the experimental task. Because the money in the task was stored in a participant's virtual wallet, we wanted to make this connection as salient as possible. Participants were told that based on their performance they would earn money to be used in the subsequent task if they answered at least three out of five of the questions correctly. However, all participants earned $\$ 50$ regardless of their performance in order to ensure experimental control. 
Next, participants completed training on Checkmate [28], then played a practice round of Checkmate with their partner. Participants were told they were randomly selected to play the Banker for the real session of five rounds and their partner was selected to play the Runner. Following the practice round, participants completed the first assessment of trust and distrust (these assessments were analyzed to test $H_{1}$ ). Each round lasted approximately three to five minutes. Following each round, participants were asked to complete other surveys that are beyond the scope of this manuscript. After the competition of the fifth experimental round, participants completed a second and final assessment of trust and distrust toward their partner (these assessments were analyzed to test $H_{2}$ and $R Q_{1}$ ). Then, participants were debriefed and paid for their time with a $\$ 30$ gift card. The money in their virtual wallet was paid to them in cash.

\section{Results}

Hypothesis 1 predicted that trust and distrust would be significantly, negatively correlated during an initial interaction, prior to a trust manipulation, between unfamiliar dyads in a trust game. We found support for this hypothesis in that trust and distrust were significantly, negatively correlated early in the experiment $(r=-.49, p<.01)$. Thus, participants who reported that they trusted their partner in the game also reported decreased distrust in their partner. In total, trust scores explained approximately $25 \%$ of the variance in distrust scores in early interactions of a trust game, prior to a trust manipulation (i.e., Trust or Distrust).

Hypothesis 2 stated that trust and distrust scores would be significantly, negatively correlated following a trust manipulation. In accordance with our expectations, trust and distrust scores were highly, negatively related later in the experiment $(r=-.75, p<$ $.01)$. That is, over $50 \%$ of the variance in the trust scores can be attributed to participants' distrust scores. The more robust correlation between trust and distrust at the end of the experiment may signify that as people interact, trustors garner more information about trustees, strengthening their attributions toward the referent (i.e., their (dis)trust toward their partner).

Finally, we examined the effects of the Runner behavior manipulation on measures of trust and distrust. That is, we conducted two independentsamples $t$-tests to examine the effect sizes (i.e., Cohen's $d$ ) of a trust violation on participants' intentions to (dis)trust their partner in future iterations of the checkmate protocol. The results showed that there was a medium effect [31] of Runner behavior on self-reported trust, $[t(62)=2.43, d=0.60]$, with those in the Trust condition $(M=3.04, S D=0.81)$ reporting higher levels of trust compared to those in the Distrust condition $(M=2.53, S D=0.86)$. In comparison, the results showed a medium to large effect [31] of the Runner behavior factor on self-reported distrust, $[t(62)$ $=3.10, d=.77]$, with participants assigned to the Distrust condition $(M=3.05, S D=0.95)$ reporting higher levels of distrust than those assigned to the Trust condition $(M=2.37, S D=0.79)$. Thus, there was a modest effect size difference $(\Delta d=.17)$ of Runner behavior between reported trust and distrust. However, based on the strong negative correlation between trust and distrust, we speculate this modest difference is a result of scale attributes (e.g., item wording) rather than evidence of for two unique constructs.

\section{Discussion}

The aforementioned theoretical models of trust assume that trust necessitates a willingness to accept some degree of vulnerability within an interaction [2; 8]. Specifically, for trust to be relevant, a trustor must experience some kind of risk in their dealings with a trustee (i.e., something must be at stake). The models of trust proposed by [2] and [8] conceptualize trust differently. Specifically, [2] (and later [11]) propose that trust spans a continuum, with trust at one end and distrust at the other. Distrust is assumed to be the absence of trust and therefore composes one pole of the trust continuum. In contrast, [8] propose that trust and distrust are orthogonal; low trust is not the same as high distrust and should therefore be considered unique criterion, similar to ambivalence toward a referent in neighboring literatures (e.g., [21]).

The present research aimed to investigate which of these two models $[2 ; 8]$ is more accurate and practically relevant. In an experimental context, trust was manipulated between subjects, and self-reported trust and distrust were assessed before and after manipulations took place. The results from the present research show that before a trust manipulation occurs, self-reported trust and distrust are negatively correlated. However, the correlation does not meet the conventional threshold in which constructs are thought to be the same (i.e., $r=.70$; [32]). After the trust manipulation occurred, these scores were negatively correlated, and this correlation was significant. The effect sizes attributed to the trust manipulation appear to be slightly larger on distrust compared to trust scores (as noted above), yet the effects appear to occur in a way such that the Trust condition led to higher trust scores than distrust scores, and the Distrust condition led to higher distrust scores than trust scores. All said, participants who reported increased trust 
toward their partner also reported decreased distrust toward their partner, and participants who reported increased distrust also reported decreased trust toward their partner. This shows evidence that increased trust corresponds to decreased distrust (and vice versa), supporting the theoretical postulates of [2] and [11] and not those of [8]. Based on these data, trust spans a continuum with trust at one end and a complete lack thereof (or distrust) at the other, evidencing one construct with two poles and variability between each end [11].

It should be noted that once participants interacted with one another over the course of the experiment, the assessments of trust and distrust correlated above the .70 mark. Such a criteria is often used in assessment contexts to indicate that two scales are really assessing the same underlying construct [32]. The experimental task served to bolster perceived risk, which may explain the increased effect size when self-reports were assessed after the manipulations. This aligns with the assumption that trust is relevant when something is at risk and hence there is a reason to be vulnerable. Additionally, as participants interacted with their confederate partner, they garnered more information used to strengthen their attributions. This may explain the comparatively modest correlation between trust and distrust at the beginning of the experiment. If parties have yet to garner much information to be used in making their attributions of one another, then it is likely they are less certain whether their partner is trustworthy or untrustworthy rather than experiencing ambivalence (i.e., experiencing both attributions of trust and distrust simultaneously) as [8] would postulate. Put simply: people do not have strong attributions of trust and distrust toward a referent before interacting, leading to a rather modest correlation between measures of trust and distrust. After interacting and garnering information about a referent (being trustworthy or untrustworthy), people can make stronger attributions toward a referent's trustworthiness. In these latter cases, researchers have an opportunity to investigate whether measures of trust and distrust span a continuum without limited interaction tempering the relationship between measures.

It should be noted that [8] did mention that although they postulated that trust and distrust were orthogonal, the relationship between the two constructs should be investigated in future work. Mittendorf [26] found that trust and distrust emerged as separate constructs; however, in addition to the aforementioned limitations of the items [26] leveraged (see section 2.2 of this paper), participants may have interacted with rideshare drivers over the years and may be conflicted in providing a single response which encompasses their experiences. Additionally, ambivalence in terms of trusting and distrusting a referent simultaneously may actually reflect a perception toward one characteristic of a referent (e.g., driver's driving ability) compared to another (e.g., driver's timeliness). The ambiguity in terms of trust and distrust may be due to not having enough information regarding the referent or characteristics of that referent. In our study, the referent was clear (partner in the task) and the context was clear (maze running task), making it easier for participants to make a trust/distrust decision.

We offer the findings that trust and distrust have a strong negative correlation. Perceptions of trustworthiness fluctuate along a continuum depending on the focal feature a trustor considers in the referent. This is something that [2] have argued, and it may be that the postulates of [8] align with [2] if one considers ambivalence to be, rather, varying trustworthiness perceptions per referent characteristics. For example, a professor (trustor) may perceive a colleague (trustee) to cover their statistics class but not give their proceedings talk at a conference. What [8] consider to be ambivalence - the presence of both trust and distrust-is simply the trustor perceiving that the trustee is competent in their statistical abilities but not in, say, their public speaking (for a similar perspective, see [33]). When provided with more context in the trust scenario (e.g., trusting someone in a certain task), the ambiguity between trust and distrust seems to abate; this was demonstrated in our study. Prior to the trust manipulation, a negative correlation between trust and distrust was found ( $r=$ $.49, p<.01$ ); at this time, participants had little information about their partner and were still familiarizing themselves with the task. Conversely, at the conclusion of the task, when participants were able to gather more information about their partner and the task at hand, the negative correlation between trust and distrust became stronger $(r=-.75, p<.01)$.

\subsection{Limitations and Future Directions}

Prior to the experimental task, participants were asked self-report questions about their partner related to trust and distrust. The measure of trust had a reliability of .60 , and the measure of distrust had a reliability of .80 . However, by the end of the experiment, reliabilities had improved to .70 for trust and .95 for distrust. One possible reason that these measures had lower reliabilities in the beginning of the experiment is because participants were unfamiliar with their partners. Theoretically, the participants would not know how to reliably answer questions such as, "I would be comfortable giving the runner a task or 
problem which was critical to me, even if I could not monitor their actions" [27] or "The runner will break rules when they have the chance" [30]. Previous research [20] has included a "Don't know" response option in addition to response options ranging from 1 (not at all) to 5 (a great deal) when measuring trustworthiness perceptions because participants may not have formed those perceptions prior to extended interactions. Future research may want to provide a similar response option on scales that measure perceptions of others, especially if data are collected prior to interactions. In addition, self-report measures of trust (and distrust) were taken only twice: once before the experiment and once at the end of the experiment. Future studies may wish to assess selfreported trust over many time periods to investigate the development of trust over time, its decay when violated, and its potential recovery.

Lastly, the distrust measure was adapted from a scale measuring general distrust toward others [30]. This scale comprises items which focus on aspects of the referent's character. These items share overlap with [2]'s conceptualization of trustworthiness perceptions, particularly integrity and benevolence. Whereas the [27]'s scale used in the present study focused mainly on the trustor's willingness to be vulnerable to the referent, the adapted [30] scale may have focused more so on the trustor's perceptions of the referent. Theoretically, however, a trustor's propensity to trust is their general willingness to accept vulnerability toward others, i.e., their general willingness to trust others. Through the lens of [2]'s model of trust - and later that of [11] — the general willingness to trust spans the same continuum with one's willingness to distrust others. Thus, we adapted a trait-based measure to reference the state-based context to assess distrust. Regardless, the items from the [30] adapted scale may assess trustworthiness rather than trust, and future research may wish to replicate and possibly extend the present findings by assessing a different measure of distrust.

\subsection{Implications}

Practically speaking, the assessments of trust accounted for roughly a quarter of the variance in the distrust assessment prior to interactions in the task. Prior to gleaning substantive information about the referent, each measure (trust and distrust) still comprised a substantive proportion of overlapping variance. After all experimental interactions, the trustor was able to glean more information about the referent's behavior and thus further solidified their perceptions of the referent's trustworthiness and ultimately their willingness to be vulnerable to the referent. Hence, the correlation between both measures was stronger, and each measure comprised over $50 \%$ of the variance explained by the other. Based on these findings, we contend that it may be the most practical method to simply assess trust. Both researchers and practitioners can benefit from reducing the number of self-report items they wish their samples to answer, and the findings from this experiment show that limiting self-reported assessments of trust may be practically informative at assessing individuals' willingness to be vulnerable to another instead of assessing the same construct with two separate measures. Using one measure potentially reduces a biased interpretation of one's results - here, lower correlations between trust and distrust at the beginning of an interchange being interpreted as trust and distrust being separate constructs - particularly at the beginning of an interchange where parties are still garnering information about one another. In a world where distributed, collaborative work is increasingly becoming the new norm, researchers interested in assessing trust between parties ought to employ the most cost-effective methods to do so. Our research provides additional support for the postulates of [2] and [11] such that trust and distrust fall on a continuum. We do not outright reject the considerations posed by [8] but reframe their interpretation as being more in line with [11] discussion of specificity when making attributions toward a referent's attributes (see also [33]). We believe that differences between trust and distrust are due to trustworthiness perceptions of different characteristics of a referent.

\subsection{Conclusion}

The present research investigated whether trust and distrust are more practically represented as two sides to a single continuum or are orthogonal constructs. Based on robust correlations and similar effects of experimental manipulations on trust and distrust criterion, there is evidence that self-reported measures of trust and distrust may be assessing the same underlying construct. Theoretically, this finding supports the postulates of [2] and [11], such that trust spans a continuum with trust at one end and distrust at another. Researchers may wish to assess a measure of trust rather than concern themselves with multiple self-report measures of the same construct.

\section{References}

[1] I. Thielmann and B.E. Hilbig, "Trust: An Integrative Review from a Person-Situation Perspective”, Review 
of General Psychology, SAGE Publications, California, United States, 2015, pp. 249-277.

[2] R.C. Mayer, J.H. Davis, and F.D. Schoorman, "An Integrative Model of Organizational Trust", Academy of Management Review, Academy of Management, United States, 1995, pp. 709-734.

[3] J.A. Colquitt, B.A. Scott, and J.A. LePine, "Trust, Trustworthiness, and Trust Propensity: A MetaAnalytic Test of their Unique Relationships with Risk Taking and Job Performance", Journal of Applied Psychology, American Psychological Association, United States, 2007, pp. 909-927.

[4] B.A De Jong, K.T. Dirks, and N. Gillespie, "Trust and Team Performance: A Meta-Analysis of Main Effects, Moderators, and Covariates", Journal of Applied Psychology, American Psychological Association, United States, 2016, pp. 1134-1150.

[5] S.L. Jarvenpaa, K. Knoll, and D.E. Leidner, "Is Anybody out There? Antecedents of Trust in Global Virtual Teams", Journal of Management Information Systems, Taylor \& Francis, United Kingdom, 1998, pp. 29-64.

[6] J.D. Lee and K.A. See, "Trust in Automation: Designing for Appropriate Reliance", Human Factors, SAGE Publications, California, United States, 2004, pp. 5080.

[7] C.S. Calhoun, P. Bobko, J.J Gallimore, and J.B. Lyons, "Linking Precursors of Interpersonal Trust to HumanAutomation Trust: An Expanded Typology and Exploratory Experiment", Journal of Trust Research, Routledge, United Kingdom, 2019, pp. 28-46.

[8] R.J. Lewicki, D.J. McAllister, and R.J. Bies, "Trust and Distrust: New Relationships and Realities", Academy of Management Review, Academy of Management, United States, 1998, pp. 438-458.

[9] D. Watson, and A. Tellegen, "Toward a Consensual Structure of Mood", Psychological Bulletin, American Psychological Association, United States, 1985, pp. 219-235.

[10] J.T. Cacioppo, and G.G. Berntson, "Relationship Between Attitudes and Evaluative Space: A Critical Review, with Emphasis on the Separability of Positive and Negative Substrates", Psychological Bulletin, American Psychological Association, United States, 1994, pp. 401-423.

[11] F.D. Schoorman, R.C. Mayer, and J.H. Davis, “An Integrative Model of Organizational Trust: Past, Present, and Future", The Academy of Management Review, Academy of Management, United States, 2007, pp. 344-354.

[12] A. Capiola, S.A. Jessup, T.J. Ryan, and G.M. Alarcon, "Exploring the Unique and Shared Variance of Propensity to Trust and Suspicion Propensity", Journal of Individual Differences, Hogrefe Publishing Group, Boston, United States, 2019, pp. 213-266.

[13] M.N. Saunders, G. Dietz, and A. Thornhill, "Trust and Distrust: Polar Opposites, or Independent but $\mathrm{Co}$ Existing?", Human Relations, SAGE Publications, California, United States, 2014, pp. 639-665.

[14] Y.T. Hung, A.R. Dennis, and L. Robert, "Trust in Virtual Teams: Towards an Integrative Model of Trust Formation", In 37th Annual Hawaii International
Conference on System Sciences, IEEE, New York, United States, 2004. pp. 1-11.

[15] L.P. Robert, A.R. Denis, and Y.T. Hung, "Individual Swift Trust and Knowledge-Based Trust in Face-toface and Virtual Team Members", Journal of Management Information Systems, Taylor \& Francis, United Kingdom 2009, pp. 241-279.

[16] A. Capiola, G.M. Alarcon, J.B. Lyons, T.J. Ryan, and T.R. Schneider, "Collective Efficacy as a Mediator of the Trustworthiness - Performance Relationship in Computer-mediated Team-based Contexts", The Journal of Psychology: Interdisciplinary and Applied, Taylor \& Francis, United Kingdom, 2019, pp. 732-757.

[17] C.B. Crisp and S.L. Jarvenpaa, "Swift Trust in Global Virtual Teams", Journal of Personnel Psychology, Wiley, New York, United States, 2013, pp. 45-56.

[18] A. Capiola, H.C. Baxter, M.D. Pfahler, C.S. Calhoun, P. Bobko, "Swift Trust in Ad Hoc Teams: A Cognitive Task Analysis of Intelligence Operators in MultiDomain Command and Control Contexts", Journal of Cognitive Engineering and Decision Making, SAGE Publications, California, United States, 2020, pp. 218241.

[19] G.M. Alarcon, J.B. Lyons, and J.C. Christensen, "The Effect of Propensity to Trust and Familiarity on Perceptions of Trustworthiness Over Time", Personality and Individual Differences, Elsevier, Netherlands, 2016, pp. 309-315.

[20] S.L. Jones and P.P. Shah, "Diagnosing the Locus of Trust: A Temporal Perspective for Trustor, Trustee, and Dyadic Influences on Perceived Trustworthiness", Journal of Applied Psychology, American Psychological Association, United States, 2016, pp. 392-414.

[21] R.E. Petty and J.T. Cacioppo, "Addressing Disturbing and Disturbed Consumer Behavior: Is it Necessary to Change the Way We Conduct Behavioral Science?", Journal of Marketing Research, SAGE Publications, California, United States, 1996, pp. 1-8.

[22] I. Katz, and R.G. Hass, "Racial Ambivalence and American Value Conflict: Correlational and Priming Studies of Dual Cognitive Structures", Journal of Personality and Social Psychology, American Psychological Association, United States, 1988, pp. 893-905.

[23] F. Herzberg, B. Mausner, and B.B. Snyderman, "The Motivation to Work (2nd ed.)", Wiley, New York, United States, 1967.

[24] J.B. Lyons, C.K. Stokes, K.J. Eschleman, G.M. Alarcon, and A.J. Barelka, "Trustworthiness and IT Suspicion: An Evaluation of the Nomological Network", Human Factors, SAGE Publications, California, United States, 2011, pp. 219-229.

[25] D.H. McKnight and V. Choudhury, "Distrust and Trust in B2C E-Commerce: Do they differ?", In Proceedings of the 8th International Conference on Electronic Commerce: The New E-Commerce: Innovations for Conquering Current Barriers, Obstacles and Limitations to Conducting Successful Business on the Internet, IEEE, New York, United States, 2006, pp. 482-491. 
[26] C. Mittendorf, "Trust and Distrust in Two-Sided Markets: An Example in the Sharing Economy", In Proceedings of the 51st Hawaii International Conference on System Sciences, IEEE, New York, United States, 2018, pp. 5402-5411.

[27] R.C. Mayer, and J.H. Davis, "The Effect of the Performance Appraisal System on Trust for Management: A Field Quasi-Experiment", Journal of Applied Psychology, American Psychological Association, United States, 1999, pp. 123.

[28] G.M. Alarcon, J.B. Lyons, J.C. Christensen, S.L. Klosterman, M.A. Bowers, T.J. Ryan, S.A. Jessup, and K.T. Wynne, "The Effect of Propensity to Trust and Perceptions of Trustworthiness on Trust in Dyads", Behavior Research Methods, Springer, New York, United States, 2018, pp. 1906-1920.

[29] J. Berg, J. Dickhaut, and K. McCabe, "Trust, Reciprocity, and Social History", Games and Economic Behavior, Elsevier, Netherlands, 1995, pp. 122-142

[30] M. Mooijman, W.W. van Dijk, N. Ellemers, and E. van Dijk, "Why Leaders Punish: A Power Perspective", Journal of Personality and Social Psychology, American Psychological Association, United States, 109, pp. 75-89.

[31] J. Cohen, "Statistical Power Analysis for The Behavioral Sciences", Second Edition, Lawrence Erlbaum Associates Publishers, Hillsdale, NJ, 1988.

[32] L.S. Fidell and B.G. Tabachnick, "Preparatory Data Analysis, In J.A. Schinka and W.F. Velicer (Eds.), Handbook of Psychology: Research Methods in Psychology", John Wiley, New York, United States, 2003, pp. 115-141.

[33] J.A. Colquitt, J.A. LePine, C.P. Zapata, and R.E. Wild, "Trust in Typical and High-Reliability Contexts: Building and Reacting to Trust Among Firefighters", Academy of Management Journal, Academy of Management, United States, 2011, pp. 999-1015. 